\title{
Development of the Pattern of Photoreceptors in the Chick Retina
}

\author{
Suzanne L. Bruhn and Constance L. Cepko \\ Department of Genetics, Harvard Medical School, Boston, Massachusetts 02115
}

The various classes of photoreceptor cells found in vertebrate retinae are organized in specific patterns, which are important for visual function. It is not known how these patterns are achieved during development. The chick retina provides an excellent model system in which to investigate this issue, containing cone opsins red, green, blue, and violet, as well as the rod-specific opsin rhodopsin. In this study, whole-mount in situ hybridization has revealed striking differences among opsins in both spatial and temporal aspects of expression. The longwavelength cone opsins, red and green, were first detected in a small spot within the area centralis at embryonic day 14 (E14). In contrast, the short-wavelength cone opsins, blue and violet, were not detected until $2 \mathrm{~d}$ later and showed domains of expression both within the area centralis and in temporal retina. The first rhodopsin transcripts were seen at E15 in inferior retina. When opsin expression was first detected, there were differences in the localization of RNA within the inner segment of cone photoreceptors, suggesting that morphological differentiation preceded the expression of photopigment molecules. Marked differences in the distribution of rods and cones were also found. Within the area centralis, a circular rod-free zone bisected by a narrow rod-sparse region along the nasal-temporal axis was evident as soon as rhodopsin RNA could be detected. Such specialized regions appear to be set aside soon after photoreceptor cells become postmitotic, as evidenced by a spatially restricted pattern of visinin RNA observed at E7. The onset of particular opsins in restricted regions of the retina suggest an underlying pattern related to visual function in the chick.

Key words: photoreceptors; rods; cones; development; chick; photoreceptor mosaic
The retina is a highly specialized sheet of neural tissue which converts photons into electrical and chemical signals that are processed by complex neural networks and translated into a coded message of action potentials to be sent to the brain. The threedimensional array of cells and cell types within the retina determines how information within the visual field is processed and perceived by the observer. Within the retina there are three stratified layers of neurons separated by two layers of synaptic connections. In an orthogonal, or tangential plane across the surface of the retina, cells within these layers are organized into patterns that allow for specialization of the visual field.

In a process analogous to that seen for pattern formation in other parts of the embryo, a two-dimensional coordinate system appears to be set up relatively early, before optic cup formation (Crossland et al., 1974; Dutting and Thanos, 1995). Morphologically, notches have been described that delineate nasal, dorsal, and temporal positions early in development and suggest that the retina shows segmentation patterns analogous to other parts of the CNS (Nordquist and McLoon, 1991). The restricted expression patterns of segmentation genes Pax2 ventrally (Nornes et al., 1990) and Wnt-7b dorsally (Parr et al., 1993) support this hypothesis. In the anterior-posterior plane, the homeobox-containing gene SOHo-I is preferentially expressed in the anterior retina (Deitcher et al., 1994). Retinoic acid, known to be involved in axial patterning of the embryo, is also found in higher concentra-

Received Scpt. 15, 1995; revised Nov. 27, 1995; accepted Nov. 29, 1995

This work was supported by National Eye Institute postdoctoral fellowship F32EYO6394 to S.L.B. and a grant from the Ruth and Milton Steinbach Foundation to C.L.C. We are grateful to Donna M. Fekete, Elio Raviola, and Elizabeth F. Ryder for critical reading of this manuscript.

Correspondence should be addressed to Constance L. Cepko, Harvard Medical School, Department of Genetics, Alpert Building, 200 Longwood Avenue, Boston, MA 02115.

Copyright $(1996$ Society for Neuroscience $0270-6474 / 96 / 161430-10 \$ 05.00 / 0$ tions in ventral retina (McCaffery et al., 1992), whereas aldehyde dehydrogenase, which oxidizes retinaldehyde to retinoic acid, is confined mainly to dorsal retina (McCaffery et al., 1991). Patterning has also been studied with respect to the retinotopic map made by ganglion cell axons. Ganglion cells, the output neurons of the retina, project through the optic nerve to the tectum, where they form a topographic map. The retinotectal system has been a model for studies of positional information and axon pathfinding and targeting. The search for molecules involved in the formation of a retinotopic map has yielded a number of topographically distributed species (Cheng et al., 1995; Drescher et al., 1995) (for review, see Kaprielian and Patterson, 1994).

Patterning within the retina has also been revealed through studies of the distribution of cells within the photoreceptor layer. The specific arrangement of rods and cones can vary dramatically across the retina and is of critical importance to the visual function of an organism. A well studied example is the human fovea, the center of which is completely free of rods (Curcio et al., 1990). Cone photoreceptor subtypes, defined by expression of unique photopigment molecules that abssorb light in particular ranges of the optical spectrum, can be organized in spatially restricted patterns; examples include the lack of blue cones in the central fovea of human retina (Curcio et al., 1991) and the dorsoventral segregation of long- and shortwave cones in the mouse (Szel et al., 1992). Achieving the complex organization of cells within the photoreceptor layer is critical, because the sampling of images from the visual field is determined by their spatial relationships. How these patterns are achieved during development is currently unknown.

In this work, whole-mount in situ hybridization has been used to investigate the spatial and temporal aspects of photoreceptorspecific molecules in the chick. This technique allows for visual- 

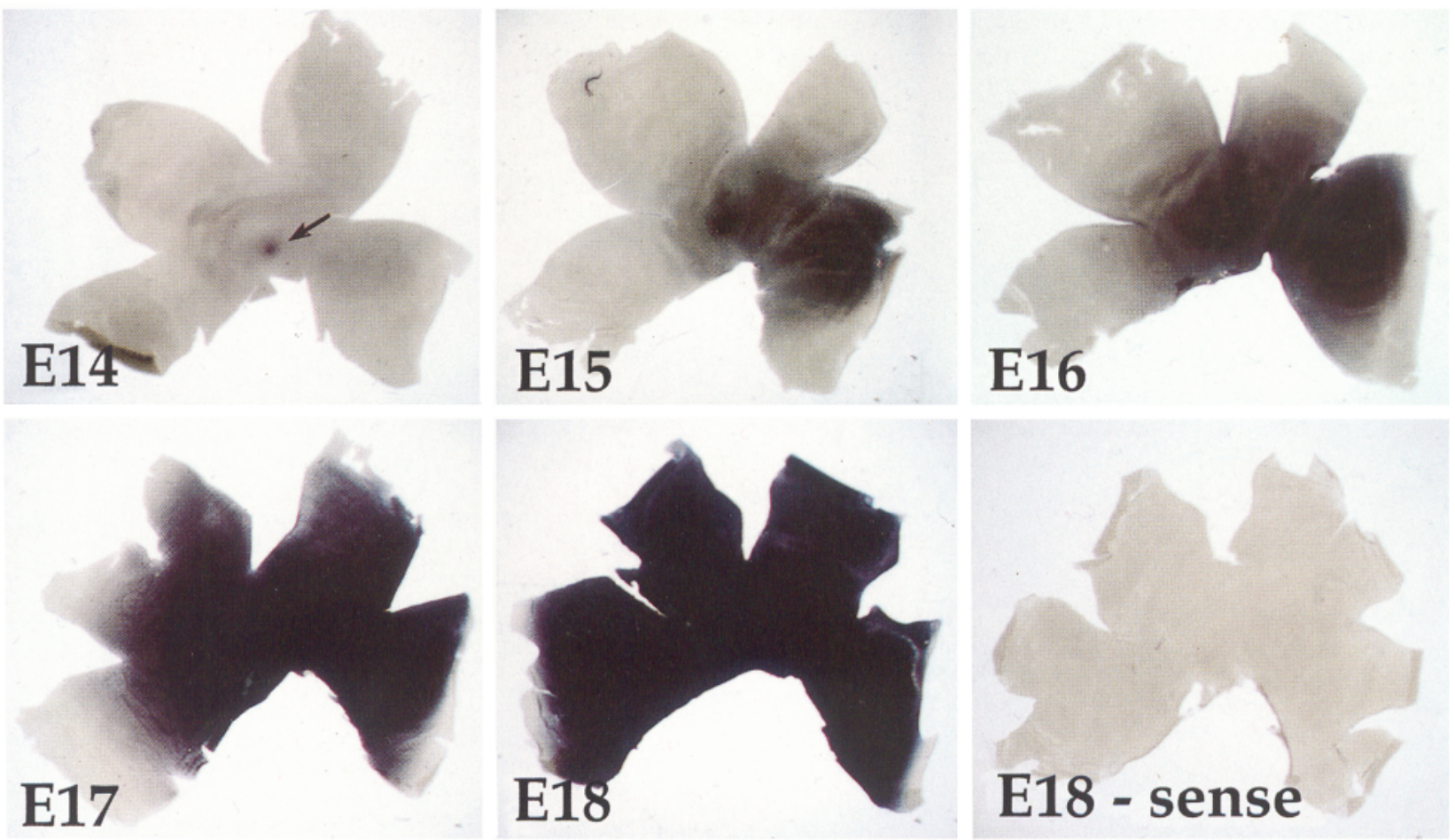

Figure 1. In the first five panels, whole-mount in situ hybridization using a digoxigenin-labeled red opsin antisense RNA probe shows the developmental time course of the expression domain. Hybridization was conducted on a series of flat-mounted retinae harvested from E14-E18. Preparation of RNA transcripts and hybridization conditions were as described in Materials and Methods. The presence of a purple formazan precipitate indicates expression of an individual opsin within a cell. The initial domain of expression at E14 is indicated by an arrow. Bottom right, Whole-mount in situ hybridization using a digoxigenin-labeled red opsin sense RNA probe on an E18 retina showing typical negligible background levels. The large cut necessary for removal of the pecten defines the inferior retina. Temporal points to the right, and nasal to the left.
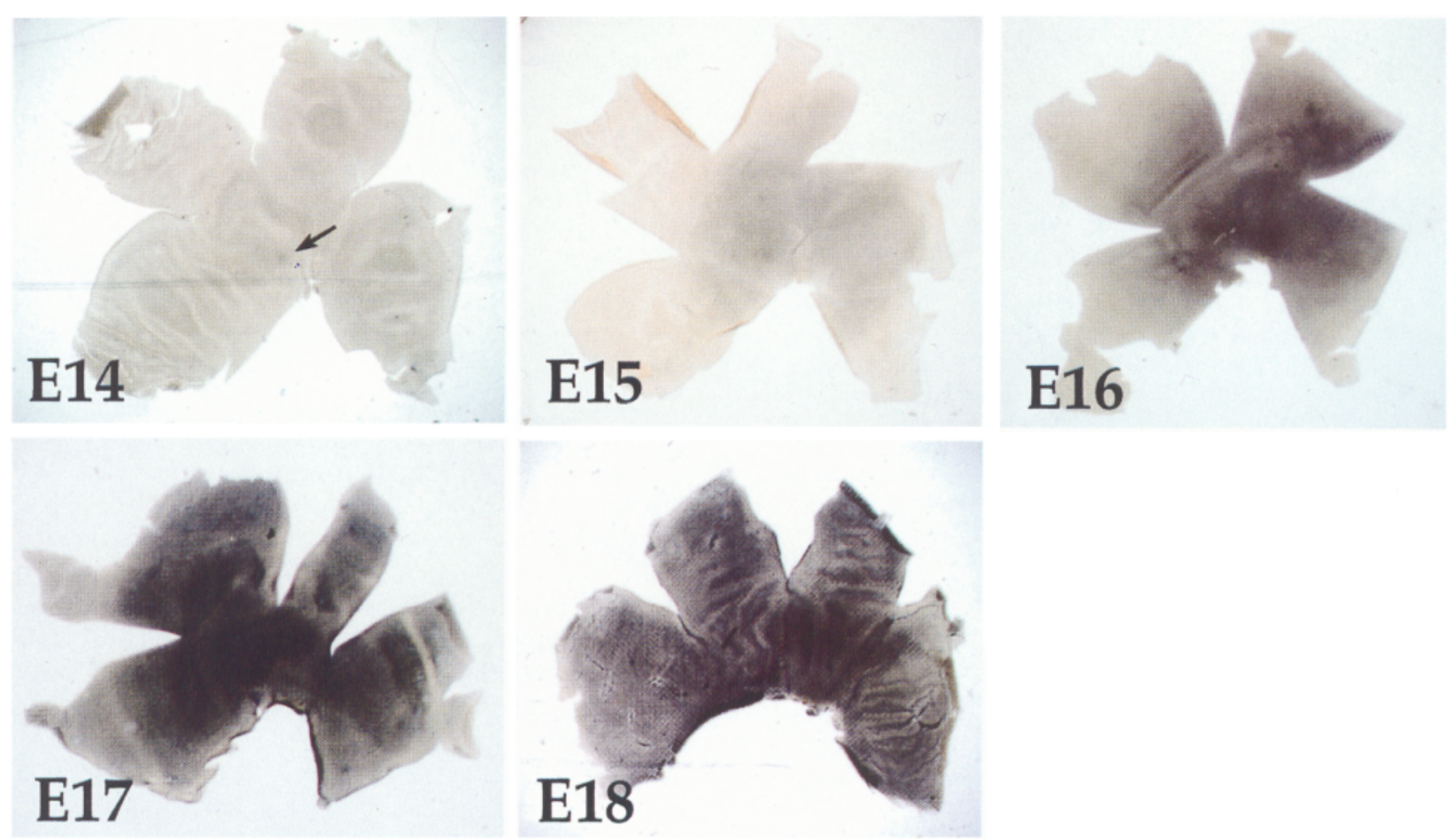

Figure 2. Results of whole-mount in situ hybridization using a digoxigenin-labeled green antisense RNA probe on flat-mounted retinae harvested from E14-E18. The region in which green opsin was first detected at E14 is indicated by an arrow. The large cut defines inferior retina. Temporal is to the right, and nasal to the left. 
ization of specific transcripts within individual cells without requiring the expression of a protein or a particular epitope, which would be necessary when using antibodies. In situ hybridization has been used previously to describe the final arrangement of photoreceptor subtypes in the goldfish and zebrafish (Raymond et al., 1993). A complete characterization of the developmental aspects of pattern formation for all photoreceptor subtypes has not been described previously in any species. The cone-rich retina of the chick provides an excellent model system in which to study multiple aspects of such development. The data presented here also provide evidence for structures amenable to the embryological and genetic manipulation of pattern. In particular, the chick retina exhibits marked differences in the distribution of rods and cones, as well as cone subtypes, which define specialized regions similar to those found in other species and which are important for visual function.

\section{MATERIALS AND METHODS}

Subcloning of chick opsins and visinin. Published sequences of chick opsin cDNAs (Takao et al., 1988; Tokunaga et al., 1990; Okano et al., 1992; Wang et al., 1992) were analyzed and aligned by computer with Genetics Computer Group software (Devereux et al., 1984) and the BLAST network service provided by the National Center for Biotechnology Information (Bethesda, MD) (Altschul et al., 1990). A 243 bp region was chosen from the (presumed) tirst exon of chicken red, green, blue, and violet opsins, and rhodopsin. This region encompasses the first two putative transmembrane domains of the proteins. In parallel, a 250 base region at the $5^{\prime}$ end of visinin cDNA sequence was also targeted for cloning (Yamagata et al., 1990). Pairs of oligonucleotides for all sequences were synthesized that contained 21 bases of complementary sequence plus a unique restriction site, either EcoRI or HindIII, at either end to facilitate subcloning. After PCR amplification of the targeted region from genomic DN $\Lambda$, fragments were digested with the appropriate enzymes and ligated into pBluescript $\operatorname{SKII}(+)$. The identities of subcloned opsins and visinin were confirmed via sequencing using standard methodology (Ausubel et al., 1989).

Harvesting of retinae. Fertilized white Leghorn chicken eggs (SPAFAS, Norwich, CT) were kept in a humidified rotating incubator at $37.5^{\circ} \mathrm{C}$ for periods up to embryonic day 18 (E18). If hatching was required, the eggs were placed in a nonrotating hatching chamber for the final 2-3 d of incubation. On the day of harvest, animals were killed, the eyes enucleated, and the retina dissected away from all surrounding tissue. A deep incision was required around the pecten to allow removal of the retina from this structure and from the pigmented epithelium. For later stages (after E16), retinae required a brief incubation in Dispase (Grade II, Boehringer Mannheim, Indianapolis, IN) to remove the pigmented epithelium completely.

Retinae were immediately fixed in a solution of $4 \% p$-formaldehyde (in PBS) overnight at $4^{\circ} \mathrm{C}$ with gentle rocking. After rinsing in PBT (PBS, $0.1 \%$ Tween-20), relinae were dehydrated in an ascending series of graded methanol for 5 min each. Retinae were stored in $100 \%$ methanol at $-20^{\circ} \mathrm{C}$ until used for in situ hybridization.

Preparation of digoxigenin-labeled riboprobes. Plasmids containing subcloned chick opsin or visinin fragments were linearized with EcoRI or HindIII and transcribed with T3 or T7 RNA polymerase for antisense or sense probes, respectively, as described previously (Riddle et al., 1993).

In situ hybridization. The in situ hybridization procedure was modified from published procedures (Riddle et al., 1993). A minimum of three retinae from each age were tested with each individual probe. At the beginning of the procedure, retinae were removed from the freezer and rehydrated through a descending series of graded methanol into PBT. After two rinses in PBT, three cuts symmetrical with the large cut at the pecten were made in each retina to allow flat mounting. Retinae were immobilized between sinall sheets of 125 min nylon mesh (Sinall Parls, Miami Lakes, FL), which were then sealed with a soldering iron.

Retinae were bleached in $6 \%$ hydrogen peroxide for $45 \mathrm{~min}$, rinsed three times, and digested for $15 \mathrm{~min}$ with $10 \mu \mathrm{g} / \mathrm{mi}$ proteinase $\mathrm{K}$. 'I'he digestion was stopped by a $10 \mathrm{~min}$ incubation in $2 \mathrm{mg} / \mathrm{ml}$ glycine, followed by two rinses in PBT. After post-fixation for $20 \mathrm{~min}$ in $4 \% \mathrm{p}$-formaldehyde $/ 0.2 \%$ glutaraldehyde, retinae were sealed into plastic bags and pretreated with hybridization solution ( $50 \%$ formamide, $5 \times \mathrm{SSC}, \mathrm{pH} 4.5$,
$50 \mu \mathrm{g} / \mathrm{ml}$ yeast RNA, $1 \%$ SDS, and $50 \mu \mathrm{g} / \mathrm{ml}$ heparin) for $1 \mathrm{hr}$ at $70^{\circ} \mathrm{C}$. Digoxigenin-labeled RNA transcript probes at $1 \mu \mathrm{g} / \mathrm{ml}$ in fresh hybridization solution were added to bags containing the retinae, and hybridization proceeded at $70^{\circ} \mathrm{C}$ overnight.

After hybridization, retinae were removed from plastic bags and rinsed three times for $30 \mathrm{~min}$ each at $70^{\circ} \mathrm{C}$ in a solution containing $50 \%$ formamide, $5 \times \mathrm{SSC}, \mathrm{pII} 4.5$, and $1 \%$ SDS. Three more $30 \mathrm{~min}$ rinses were performed at $65^{\circ} \mathrm{C}$ in a solution of $50 \%$ formamide and $2 \times \mathrm{SSC}, \mathrm{pH}$ 4.5. Before incubation with the alkaline-phosphatase-conjugated antidigoxigenin Fab antibody (Boehringer Mannheim), retinae were preblocked for $2.5 \mathrm{hr}$ in Tris-buffered saline containing $1 \%$ Tween-20 (TBST) and 10\% heat-inactivated sheep serum. Nonspecific binding was further prevented by preadsorption of the antibody in $1 \%$ sheep serum and $0.3 \%$ heat-inactivated chick embryo powder. Retinae were incubated overnight at $4^{\circ} \mathrm{C}$ in a $1: 2000$ dilution of preadsorbed antibody in TBST and $1 \%$ heat-inactivated sheep serum. To remove excess antibody, retinae were rinsed in TBST three times for 5 min at room temperature, five times for $1.5 \mathrm{hr}$ at room temperature, and overnight at $4^{\circ} \mathrm{C}$. For the antibody detection reaction, retinae were equilibrated in NTMT $(100 \mathrm{~mm}$ $\mathrm{NaCl}, 100 \mathrm{~mm}$ Tris, pH 9.5, $50 \mathrm{~mm} \mathrm{MgCl}_{2}, 0.1 \%$ Tween-20, $2 \mathrm{~mm}$ Levamisole) by three 10 min rinses. The detection reaction was performed in foil-wrapped containers with $0.25 \mathrm{mg} / \mathrm{ml}$ nitroblue tetrazolium and $0.13 \mathrm{mg} / \mathrm{ml}$ 5-bromo-4-chloro-3-indolyl phosphate in NTMT for $4 \mathrm{hr}$ at room temperature, and then overnight with fresh solutions at $4^{\circ} \mathrm{C}$. The reaction was stopped by two $10 \mathrm{~min}$ rinses in NTMT followed by a $10 \mathrm{~min}$ incubation in PBT, pH 5.5. Retinae were post-fixed in $4 \% p$-formaldehyde $/ 0.1 \%$ glutaraldehyde for $1 \mathrm{hr}$ and then rinsed twice in PBT.

After removal from the nylon mesh, flat-mounted preparations were photographed using a Nikon zoom stereo microscope with Kodak Elite 100 film (Rochester, NY). For optical sections, retinae were cleared through an ascending series of graded glycerol and mounted photoreceptor-side up in $100 \%$ glycerol. Retinae were viewed at $63 \times$ with Nomarski optics using a Zeiss Axiophot microscope (Thornwood, NY) and photographed with Kodak 64T film.

Cryostat sectioning. After in situ hybridization, retinae were infiltrated with $30 \%$ sucrose in PBS and embedded in OCT (Tissue-Tek, Miles, Elkhart, IN). Frozen sections were cut at $20 \mu \mathrm{m}$ on a Reichert Jung CM3000 cryostat (Leica, Deerfield, IL) and collected on Superfrost Plus slides (Fisher Scientific, Pittsburgh, PA). After rehydration in PBS, sections were mounted in gelvatol and coverslipped.

\section{RESULTS}

\section{Developmental pattern of cone opsin expression}

The technique of whole-mount in situ hybridization was used to investigate spatial and temporal aspects of opsin expression in the chick retina. Digoxigenin-labeled probes specific for red, green, blue, and violet opsin transcripts were hybridized to retinae harvested from E13-E18, as well as to hatchling retinae. The use of an alkaline-phosphatase-conjugated anti-digoxigenin antibody and standard histochemistry resulted in deposition of a purple formazan precipitate within each cell expressing the relevant RNA. Digoxigenin-labeled sense control probes were used for hybridization at several ages for each probe and were always completely negative (for example, see Fig. 1).

Specificity of hybridization was assured in several ways. First, riboprobes encompassed a small region $(243 \mathrm{bp})$ of the opsin molecule that showed the least homology among members of the family (ranging from 51 to $66 \%$ between pairs of sequences). Second, hybridization was performed under very stringent conditions to preclude cross-reactivity. Third, and most important, the expression patterus of individual opsin transcripts were distinct. Macroscopically, differences were seen both spatially and temporally in the expression patterns of opsins. In addition, striking differences in RNA localization were found within the inner segments of photoreceptors (see below).

Representative results of a series of whole-mount in situ hybridization experiments with digoxigenin-labeled red opsin transcripts are shown in Figure 1. The presence of red opsin transcripts was first detected at E14 in a small spot in the area 

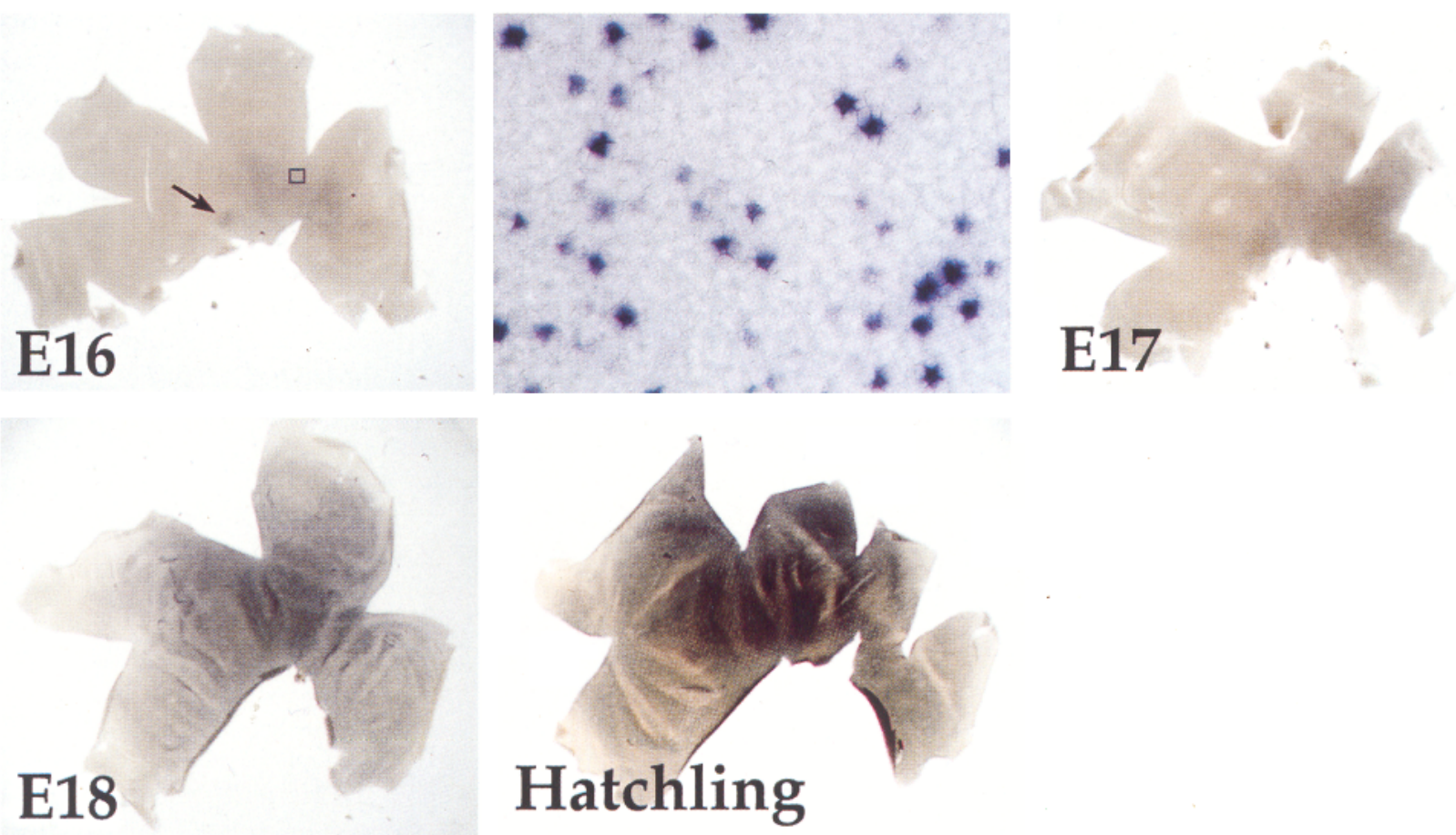

Figure 3. Results of a series of whole-mount in situ hybridizations using a digoxigenin-labeled antisense blue probe on flat-mounted retinae harvested from E16 to hatchling chicks. The initial domain of expression in the area centralis at E16 is indicated by an arrow. The boxed region from the temporal domain of expression at E16 is shown at higher power in the adjacent panel. The large cut necessary for removal of the pecten defines inferior retina. Temporal is to the right, and nasal is to the left.

centralis, slightly nasal and superior to the optic nerve head. The optic nerve exits the chick retina at the central terminus of the optic fissure, the location of which is indicated by a large cut in the inferior portion of the retina. By E15, the expression domain of red opsin had expanded in a central-to-peripheral gradient, asym- metrically biased toward temporal retina. One day later at E16, red opsin transcripts could be detected over most of the central and temporal retina. The central-to-peripheral gradient of expression continued to expand until E18, at which point red opsin transcripts were represented over the entire surface of the retina.
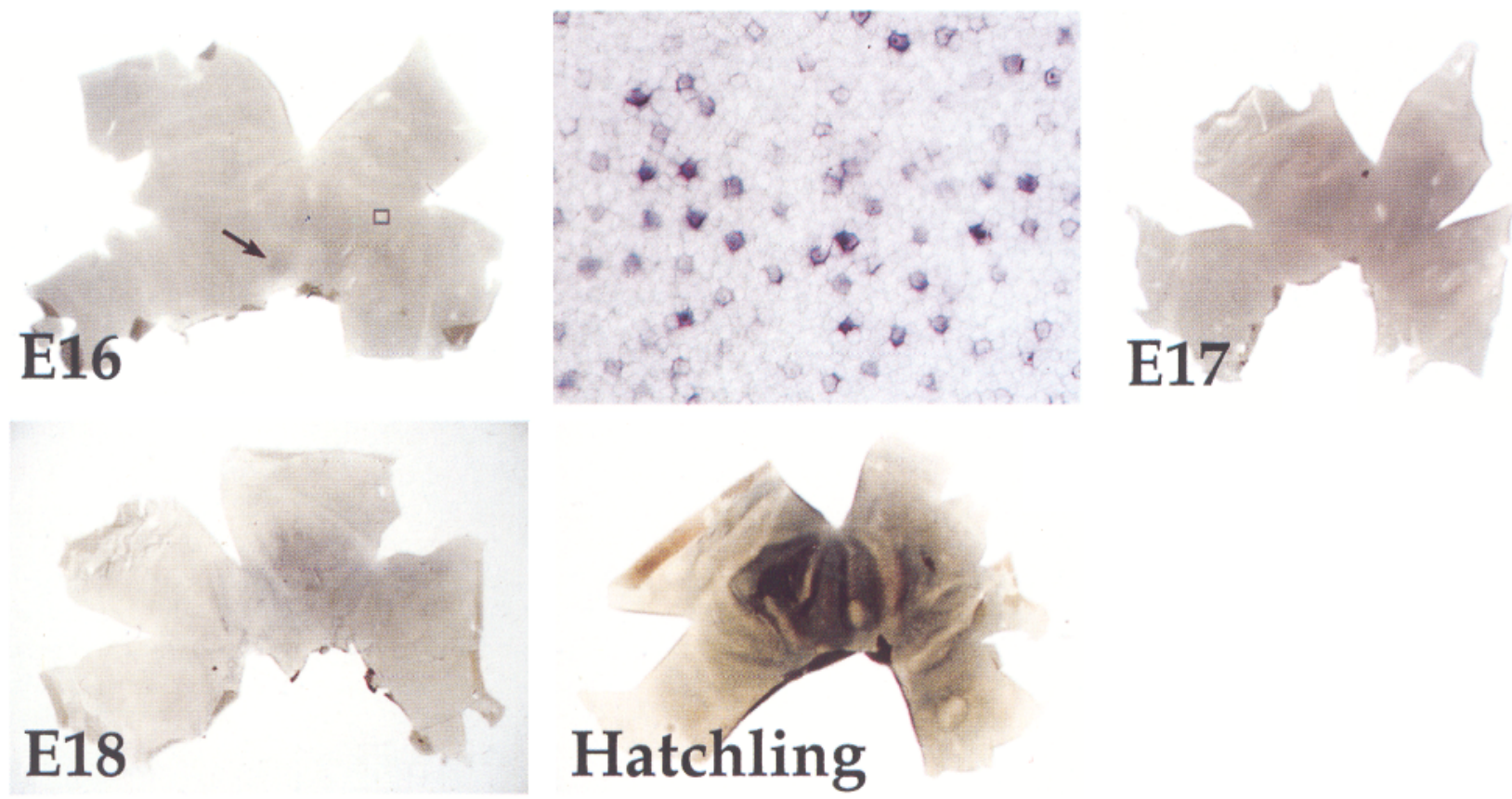

Figure 4. Results of a series of whole-mount in situ hybridizations using a digoxigenin-labeled antisense probe specific for violet opsin on flat-mounted retinae harvested from E16 to hatchling chicks. The region in which violet opsin was first detected at E16 is indicated by an arrow. The boxed region within the temporal domain of expression at $\mathrm{E} 16$ is shown in the adjacent panel at higher power. The large cut defines inferior retina. Temporal is to the right, and nasal is to the left. 
The spatial and temporal aspects of expression of green opsin RNA were identical to those of red opsin, as seen in Figure 2. The first detectable green opsin transcripts were found at E14 in an anatomically similar region of the area centralis, just nasal and superior to the optic nerve. The expression domain expanded from center to periphery with the leading edge showing a bias toward temporal retina at E15. This domain continued to expand until E18, when green opsin transcripts could be detected over the entire surface of the retina, although the relative abundance of green cones was much less than that of red cones. The intensity of signal at E18 is a function of the proportion of cells expressing the final functional opsin phenotype, confirmed via optical sections from cleared whole-mount preparations of E18 and hatchling retinae (not shown).

The chick retina has two short-wavelength photopigment molecules, blue and violet. As seen in Figures 3 and 4, both the spatial and temporal aspects of the expression domains of these two opsin transcripts differed from those of their longer wavelength counterparts. In Figure 3, it is shown that the first blue opsin transcripts were detected in two retinal locations on E16. One cluster of positive cells was located in the area centralis in a similar or identical spot to that in which red and green upsin transcripts were initially detected. Blue opsin transcripts were also detected in temporal retina; a higher magnification picture is shown in an adjacent panel because the low abundance of this photoreceptor subtype makes it difficult to visualize positive cells on the whole-mount preparations. Similarly, the first detectable violet opsin transcripts were seen on E16, originating in similar or identical anatomical positions, as delineated in Figure 4. Over the next $2 \mathrm{~d}$, the expression domains of both of these RNAs spread to more peripheral regions until they were detected across the entire retina in the hatchling chick. Cones containing violet opsin were the least abundant photoreceptor subtype within the chick retina.

\section{Developmental pattern of rhodopsin expression}

The first detectable rhodopsin transcripts were seen at E15 at quite a different location than any of the cone opsins, as shown in Figure 5. The earliest cluster of cells expressing rhodopsin was seen in inferior retina, in a region surrounding the optic fissure. Expression continued to spread peripherally over the next 2-3 d until the final pattern of rods was achieved by E18. This final pattern was in striking contrast to the cone photoreceptor subtypes, which were distributed over the entire surface of the retina. Rods were much more abundant in the inferior half of the mature retina and were completely absent from a circular region in the area centralis just nasal and superior to the optic nerve. In addition, there was a narrow rod-sparse band along the nasal-temporal axis of the retina that bisected the "rod-free" zone. Curiously, a thin rod-sparse region also was present circumferentially in peripheral retina, the location of which was not correlated to any known anatomical structures. The mature topographic pattern was evident as early as E16 and appeared to be set up quite early in the program of rhodopsin transcript expression.

\section{Opsin transcripts show differing subcellular localization}

In addition to the differing spatial and temporal patterns of expression among photoreceptor subtypes, the distribution of each class of opsin transcripts within individual cells, most notable upon first expression, was quite dissimilar. A series of optical sections at the level of the inner segment is shown in Figure 6. The day on which opsin was first detected is shown for all. At E14, a closer look at the first cells expressing red opsin within the area centralis reveals that the RNA both within and among these cells was distributed in a very diffuse pattern. In contrast, however, the cells in this same anatomical location that expressed green opsin transcripts at the same time were quite distinct. In these cells, the RNA appeared to fill the entire inner segment within a single plane of focus. At developmentally equivalent regions of the retina such as the leading edge of the domain of expression of these transcripts at E16, the patterns looked identical to those of the respective opsins at E14 in the area centralis (not shown).

The short-wavelength opsins blue and violet showed RNA localization that was distinct from those of either red or green. Figure 6 shows an optical section through the inner segments of these photoreceptors in a focal plane slightly vitreal to those shown for red and green cones. For the short-wavelength opsins, the RNA was tightly localized within a small structure in the center of the inner segment. These differences in RNA localization may reflect a different subcellular organization among the photoreceptor subtypes present at or before the first expression of their final functional opsin phenotype.

Rods first showed expression of their photopigment RNA at E15 within cells that can be anatomically differentiated from cone photoreceptor cells by their much thinner inner-segment processes (Morris, 1970). Figure 6 shows an optical section taken near the area centralis (for comparison with cone photoreceptors). Rhodopsin transcripts appeared to span the width of the cell compartment in which they were present. The sharp differences in abundance across the retina were visible as soon as the transcript could be detected.

\section{Expression of visinin transcripts is detected as soon as photoreceptors become postmitotic}

Although opsin RNA transcription was first evident at E14, classic birthdating studies have placed the birthdays of photoreceptors considerably earlier, beginning at E6 or before (Fujita and Horii, 1963; Kahn, 1974; Prada et al., 1991). To determine whether other aspects of cone differentiation occurred before opsin synthesis, in situ hybridization was performed using a probe specific for visinin. Visinin is a small calcium-hinding protein, which was initially reported to be an early and specific marker of photoreceptor cells in the chick (Hatakenaka et al., 1985; Yamagata et al., 1990). As seen in Figure 7, visinin transcripts were observed in flat-mounted preparations as early as E6 in a small spot near the center of the developing retina. Expression followed the course of mitotic activity, i.e., in a central-to-peripheral gradient. At E7, the pattern of visinin-expressing cells already showed characteristics of the regional specialization seen in the pattern of mature photoreceptors, i.e., areas that may be set aside for differing photoreceptor distributions in the area centralis, along the nasal-temporal axis, and circumferentially in peripheral retina. These regions are reminiscent of the circular rod-free zone and narrow rod-sparse band revealed by rhodopsin hybridization, but do not reflect the superior/inferior gradient of rods present in the mature retina. By E8, visinin RNA could be detected across the entire developing retina. Curiously, higher levels of expression were seen in peripheral retina at this age. Later in development, high levels of RNA were distributed evenly across the retina (not shown).

Sectioning of flat-mounted preparations revealed that the visinin transcripts were localized within the cells of the presumptive photoreceptor layer at these early stages of retinal development. At E6, when the first photoreceptor cells become postmitotic 

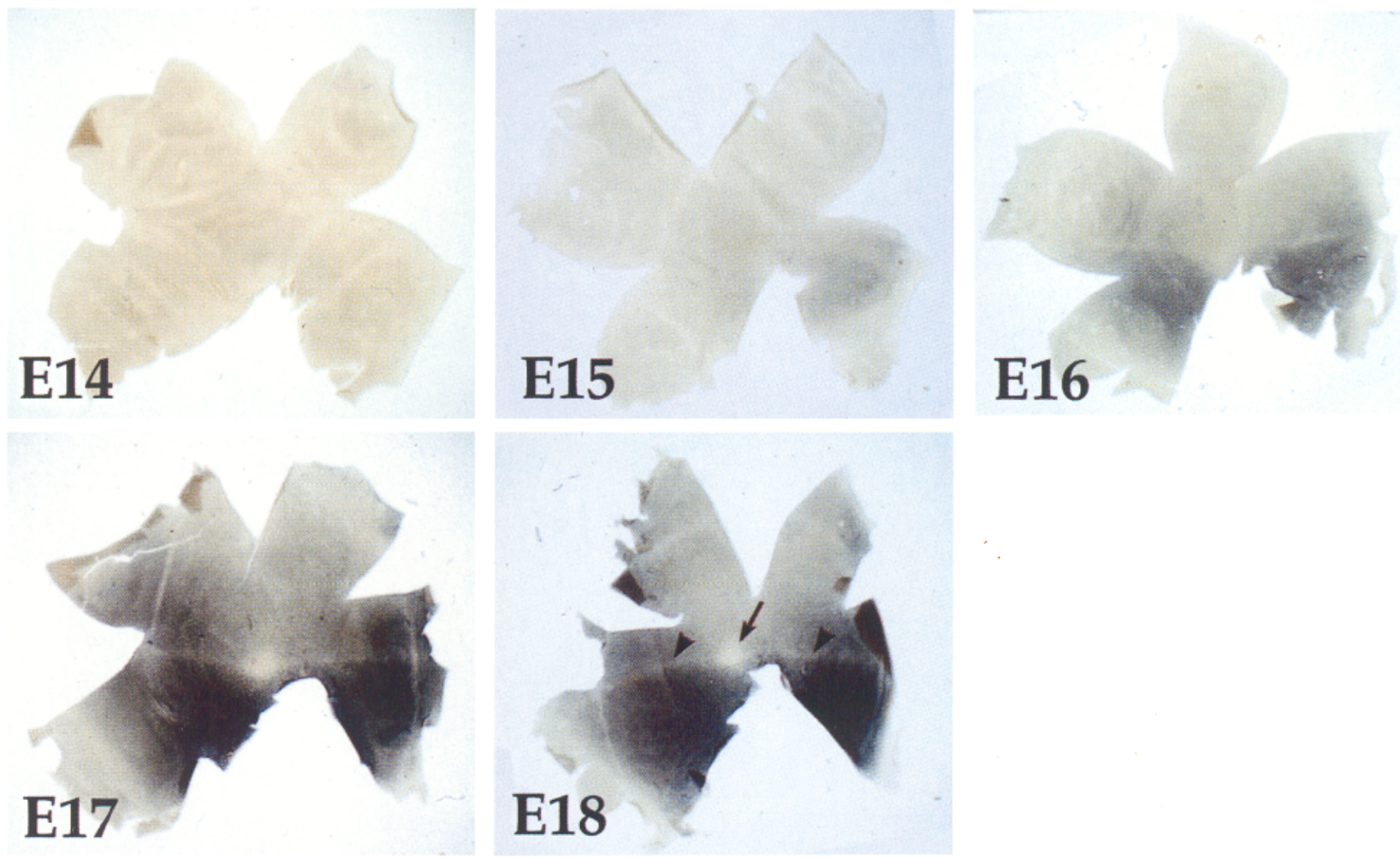

Figure 5. Results of a series of whole-mount in situ hybridizations using a digoxigenin-labeled rhodopsin antisense RNA probe on flat-mounted retinae harvested from E14-E18 showing the spatial and temporal aspects of the expression domain. At E18, the arrow denotes the rod-free zone in the area centralis. Arrowheads point to the narrow rod-sparse band along the nasal-temporal axis. The large cut necessary for removal of the pecten defines the inferior retina. Temporal is to the right, and nasal is to the left.

(Fujita and Horii, 1963; Kahn, 1974; Prada et al., 1991), visinin was expressed in small clusters of cells on the scleral side of the retina. The size of each cluster of positive cells increased until E8, when the visinin-expressing cells formed a band at what will eventually become the photoreceptor layer.

\section{DISCUSSION}

Differential regional distributions of the two major classes of photoreceptors, rods and cones, can subserve specialized retinal functions within the visual field in dim or bright light. The results presented here reveal that such areas do exist within the chick retina and that these patterns are evident at the time of initial expression of opsin RNA. Specifically, there is a circular rod-free zone within the area centralis bisected by a narrow rod-sparse band along the nasal-temporal axis, which allows for a higher relative number of cones and presumably higher visual acuity in these portions of the visual field. Transcripts of all four cone photopigment subtypes were detected in the rod-free portion of the area centralis, indicating that the chick can absorb light under photopic conditions from across the visible spectrum in this specialized region. This area correlates anatomically with the region of highest ganglion cell density in the chick (Ehrlich, 1981; Straznicky and Chehade, 1987) and with a specialized region within the inner nuclear layer termed the aster, where cells are arranged in a centrally symmetric, outwardly radiating pattern (Morris, 1982). Specialization within other layers of the retina at this anatomical position supports the hypothesis that the formation of the pattern within this region has significance for visual function in the chick.

Little is known about the kinetics and mechanisms of the formation of such patterns within the photoreceptor layer of the retina. In the chick, the first rhodopsin transcripts were detected at $\mathrm{E} 15$ in the inferior half of the retina in a distribution that already reflected the final superior-to-inferior gradient of rods found in mature retina. By E16, when rhodopsin was first expressed in the cells of the central retina, the final pattern with respect to the rod-free and rod-sparse areas was apparent. Similarly, in primates, the fovea appears to be specified very early, with the final photoreceptor composition evident as soon as the region is identifiable (Hendrickson, 1992). It is possible that such specialized areas are distinct from other regions of the retina in their developmental programs. In the human retina, photoreceptors within the fovea appear by morphological criteria to be developmentally delayed with respect to more peripheral cones, despite their earlier birthdays (Hendrickson and Drucker, 1992). The curious pattern of visinin transcripts evident at E7 in the chick, which mirror aspects of the regional specialization of the mature retina, may reflect a similar developmental program or delay.

The striking spatial and temporal differences seen between rod and cone opsin transcripts during chick development suggests that these classes of photoreceptors are distinct as soon as they can be distinguished by their opsin expression. There are no markers currently available that can distinguish rods and cones before expression of class-specific phototransduction proteins. Visinin was initially reported to be an early and specific marker for cone photoreceptors in the chick, the cone homolog of the rod-specific recoverin (Hatakenaka et al., 1985; Yamagata et al., 1990). Recoverin and visinin have subsequently been found in all photoreceptor cells, however, suggesting that the differences between 

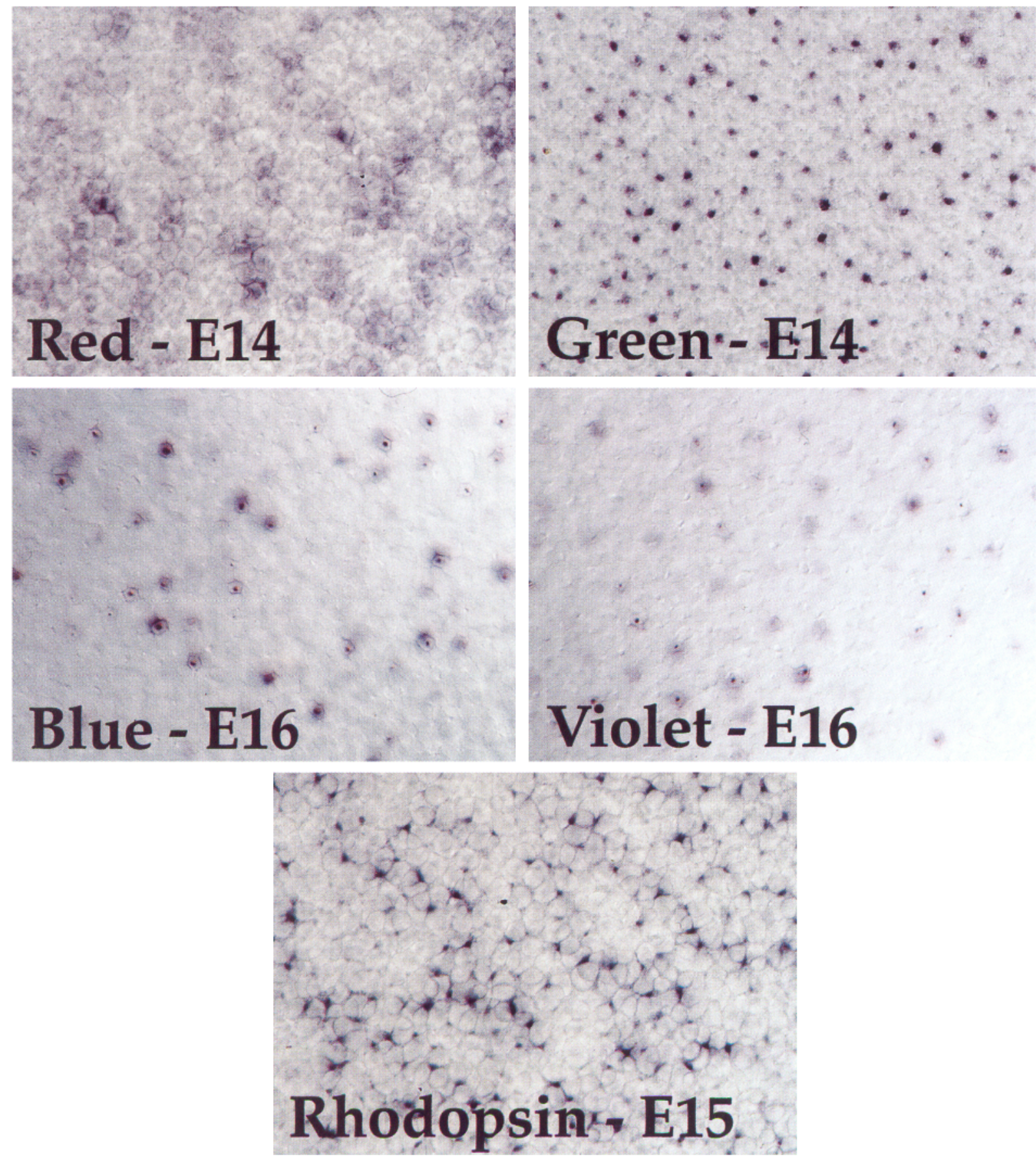

Figure 6. High-power views of cleared, flat-mounted retinae at the level of photoreceptor inner segments after whole-mount in situ hybridization. Views selected represent the initial appearance of each transcript. For red and green opsin, areas depicted are within the first site of expression in the area centralis at E14. At E16, the first blue and violet opsin transcripts can be detected; the optical section shown is from central retina. At E15, rhodopsin transcript containing cells are shown from an area near the center of the retina for comparison with cone photoreceptors.

these two proteins simply reflect the species from which they were originally isolated (Polans et al., 1993; Wiechmann and Hammarback, 1993). XAP-1, another early marker of photoreceptor fate, is expressed in all photoreceptors of the frog. The subsequent expression of XAP-2, which labels only rods, led to a model of an early, generalized photoreceptor state, which preceded the decision to become a rod or a cone (Harris and Messersmith, 1992). Birthdating studies in which rod and cone birthdays have been discriminated, such as in the primate (LaVail et al., 1991), mouse
(Carter-Dawson and LaVail, 1979; Young, 1985a,b), and chick (Morris, 1973), however, indicate that these two types of photoreceptors become postmitotic in temporally distinct patterns. It seems likely that these two major classes of photoreceptors are somehow different from birth.

Cone photoreceptor subtypes also appear to be unique in their domains of expression as soon as expression can be detected. Patterns of expression among subtypes do not reflect the overall kinetics of mitotic activity seen in the chick retina, 


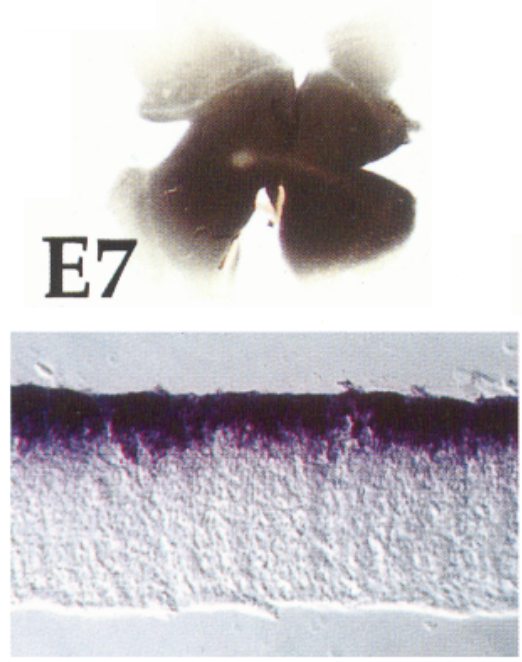


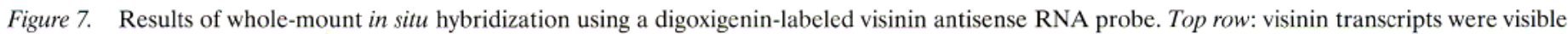
as early as E6 in the central region of the retina. By E8, the presence of this transcript could be detected over the entire surface of the retina. Bottom row: cryostat sections of material represented in panels directly above. At E6, cells expressing visinin were found in small patches along the ventricular surface. The number of cells expressing this transcript had increased by E8, and was represented by a band of cells in the presumptive photoreceptor layer.

which approximates a simple central-to-peripheral gradient across the retina (Fujita and Horii, 1963; Kahn, 1974; Dutting et al., 1983; Prada et al., 1991). Although the expression of red and green opsins was evident in the central retina at E14, blue and violet opsin transcripts were not seen there until $2 \mathrm{~d}$ later. Small differences among birthdays measured for morphological classes of cones in the chick (Morris, 1973) cannot account for the time differences seen in the appearance of RNA encoding the cone opsins seen in this study. Thus, although a population of cells within one area of the retina may become postmitotic in a temporally synchronous manner, classes of cells within that area showed differences in the time course of expression of their photopigment molecules. Curiously, the short-wavelength pigments blue and violet initiated their expression in both central and temporal retina. It is interesting to speculate whether the temporal domain of expression of the shortwave pigment transcripts reflects an evolutionary relationship to the second fovea found in the temporal retina of related avian species such as the pigeon (Rodieck, 1973).

One potentially useful marker of photoreceptor subtype differentiation is the structural differences observed among classes of rods and cones. In the chick, classic electron microscopic studies have described a number of morphological subtypes of photoreceptors, including rods, double cones, and three types of single cones arranged in a symmetrical pattern (Morris and Shorey, 1967; Morris, 1970). Whether these structural parameters correlate with the expression of particular photopigment molecules within a functional cone photoreceptor is not known, however. In one immunocytochemical study, anti-red opsin antibodies were found to label both cells of the double cone and one type of single cone (Araki et al., 1990). If further correlations could be found, these anatomical parameters would provide another useful marker of subtype identity. In zebrafish (Larison and Bremiller, 1990) and Xenopus (Saha and Grainger, 1993), opsin expression precedes morphological differentiation. In the chick, however, the subcellular compartmentalization of opsin RNAs appeared remarkably dissimilar among subtype classes as soon as they could be detected. This observation may indicate that structural differences are present before the expression of class-specific opsin molecules in the chick. Although it is not possible at the light microscope level to identify the particular structures within the inner segment to which the RNA is localized, striking differences in morphology can be seen with the electron microscope among cells of this class (Morris and Shorey, 1967). Most notably, subsets of mature photoreceptors contain an endoplasmic reticulum that is widely variant in abundance and organization (S. L. Bruhn and C. L. Cepko, unpublished observations).

Many models have been proposed to explain the program of differentiation of photoreceptor cells, which ultimately requires a complex cellular structure and the expression of specific proteins necessary for phototransduction. It is not known when the decision to express an individual pigment that can define a photoreceptor subtype occurs; it could be shortly after the cell is born or it could represent a later stage of differentiation. In species with an extended period of neurogenesis, such as that which occurs in birds (Fujita and Horii, 1963; Kahn, 1974; Prada et al., 1991), rodents (Carter-Dawson and LaVail, 1979; Young, 1985a,b), and primates (LaVail et al., 1991), classical birthdating studies suggest that there is a delay between the final mitotic division of the presumptive photoreceptor cell and expression of markers that can distinguish its ultimate fate. In the chick, for example, there is a delay of 8-10 d between the day of birth of photoreceptors and the expression of most of the markers of their differentiated phenotype. The point along this pathway at which the cell becomes irreversibly committed is unclear. The expression of visinin RNA at E6 in presumptive photoreceptor cells of the chick indicates that these cells have acquired some information regarding their fate as soon as they become postmitotic.

It seems likely that the identities and positions of photoreceptor subtypes are specified in a tightly regulated process, perhaps with a feedback mechanism. Some fine-tuning of the pattern may occur late in development via migration of differentiated cells, as has been suggested to occur in the macaque (Packer et al., 1990). In 
the mouse, blue cones precede red/green cones in the expression of their opsins, maintaining their superior-inferior segregation throughout the period of differentiation (Szel et al., 1993). However, recent studies using antibodies suggest that some proportion of cells along the border of these two regions can express both opsin molecules in the mature retina (Rohlich et al., 1994). In rat and gerbil retinae, opsin double-positive cells were detected transiently during photoreceptor development (Szel et al., 1994). Although the presence of two independent RNA molecules within a single cell would be more definitive proof of transient double expression than the presence of two antibody epitopes, which might be shared, it has proven to be technically difficult to achieve in the present system.

In the primate, the early detection of red/green opsin-specific markers within cells distributed in a regularly spaced array has led to the hypothesis that these "precocious" cones represent a protomap of the photoreceptor mosaic with instructive polential for other undifferentiated cells (Wikler and Rakic, 1991, 1994). Molecular and morphological differences among cell types seen during the process of differentiation do not directly address issues of commitment or potential, however. It is equally plausible that the temporal aspects of markers of photoreceptor cell fate reflect progressive states of differentiation along a predetermined pathway. Ultimately, the mechanism and timing of commitment decisions can only be discerned by manipulating the environment of relevant cells during development.

\section{REFERENCES}

Altschul SF, Gish W, Miller W, Myers EW, Lipman DJ (1990) Basic local alignment scarch tool. J Mol Biol 215:403-410.

Araki M, Fukada Y, Shichida Y, Yoshizawa T (1990) Localization of iodopsin in the chick retina during in vivo and in vitro cone differentiation. Invest Ophthalmol Vis Sci 31:1466-1473.

Ausubel FM, Brent R, Kingston RE, Moore DD, Seidman JG, Smith JA, Struhl K (1989) Current protocols in molecular biology. New York: Greene Publishing Associates.

Carter-Dawson LD, LaVail MM (1979) Rods and cones in the mouse retina. II. Autoradiographic analysis of cell generation using tritiated thymidine. J Comp Neurol 188:263-272.

Cheng HJ, Nakamoto M, Bergemann AD, Flanagan JG (1995) Complementary gradients in expression and binding of ELF-1 and Mek4 in development of the topographic retinotectal projection map. Cell 82:371-381

Crossland WJ, Cowan WM, Rogers LA, Kelly JP (1974) The specification of the retino-fectal projection in the chick. J Comp Neurol $155: 127-164$

Curcio CA, Sloan KR, Kalina RE, Hendrickson AE (1990) Human photoreceptor topography. J Comp Neurol 292:497-523.

Curcio CA, Allen KA, Sloan KR, Lerea CL, Hurley JB, Klock IB, Milam AH (1991) Distribution and morphology of human cone photoreceptors stained with anti-blue opsin. J Comp Neurol 312:610-624.

Deitcher DL, Fekete DM, Cepko CL (1994) Asymmetric expression of a novel homeobox gene in vertebrate sensory organs. J Neurosci $14: 486-498$.

Devereux J, Haeberli P, Smithies O (1984) A comprehensive set of sequence analysis programs for the VAX. Nucleic Acids Res 12:387-394.

Drescher U, Kremoser C, Handwerker C, Loschinger J, Noda M, Bonhoeffer $F$ (1995) In vitro guidance of retinal ganglion cell axons by RAGS, a $25 \mathrm{kDa}$ tcctal protcin related to ligands for Eph receptor tyrosine kinases. Cell 82:359-370.

Dutting D, Thanos S (1995) Early determination of nasal-temporal retinotopic specificity in the eye anlage of the chick embryo. Dev Biol 167:263-281.

Dutting D, Gierer A, Hansmann G (1983) Self-renewal of stem cells and differentiation of nerve cells in the developing chick retina. Brain Res $312: 21-32$.
Ehrlich D (1981) Regional specialization of the chick retina as revealed by the size and density of neurons in the ganglion cell layer. J Comp Neurol 195:643-657.

Fujita S, Horii M (1963) Analysis of cytogenesis in chick retina by tritiated thymidine autoradiography. Arch Histol Jpn 23:359-366.

Harris WA, Messersmith SL (1992) Two cellular inductions involved in photoreceptor determination in the Xenopus retina. Neuron 9:357-372.

Hatakenaka S, Kiyama H, Tohyama M, Miki N (1985) Immunohistochemical localization of chick retinal 24 kdalton protein (visinin) in various vertebrate retinae. Brain Res 331:209-215.

Hendrickson A (1992) A morphological comparison of foveal development in man and monkey. Eye 6:136 144.

Hendrickson A, Drucker D (1992) The development of parafoveal and mid-peripheral human retina. Behav Brain Res 49:21-31.

Kahn AJ (1974) An autoradiographic analysis of the time of appearance of neurons in the developing chick neural retina. Dev Biol 38:30-40.

Kaprielian Z, Patterson PH (1994) The molecular basis of retinotectal topography. Bioessays 16:1-11.

Larison KD, Bremiller R (1990) Early onset of phenotype and cell patterning in the embryonic zebrafish retina. Development 109:567-576.

LaVail MM, Rapaport DH, Rakic P (1991) Cytogenesis in the monkey retina. J Comp Neurol 309:86-114.

McCaffery P, Tempst P, Lara G, Drager UC (1991) Aldehyde dehydrogenase is a positional marker in the retina. Development 112:693-702.

McCaffery P, Lee MO, Wagner MA, Sladek NE, Drager UC (1992) Asymmetrical retinoic acid synthesis in the dorsoventral axis of the retina. Development 115:371-382.

Morris VB (1970) Symmetry in a receptor mosaic demonstrated in the chick from the frequencies, spacing and arrangement of the types of retinal receptor. J Comp Neurol 140:359-398.

Morris VB (1973) Time differences in the formation of the receptor types in the developing chick retina. J Comp Neurol 151:323-330.

Morris VB (1982) An afoveate area centralis in the chick retina. J Comp Neurol 210:198-203.

Morris VB, Shorey CD (1967) An electron microscope study of types of receptor in the chick retina. J Comp Neurol 129:313-340.

Nordquist D, McLoon SC (1991) Morphological patterns in the developing vertebrate retina. Anat Embryol (Berl) 184:433-440.

Nornes HO, Dressler GR, Knapik EW, Deutsch U, Gruss P (1990) Spatially and temporally restricted expression of Pax2 during murine neurogenesis. Development 109:797-809.

Okano T, Kojima D, Fukada Y, Shichida Y, Yoshizawa T' (1992) Primary structures of chicken cone visual pigments: vertebrate rhodopsins have evolved out of cone visual pigments. Proc Natl Acad Sci USA 89:5932-5936.

Packer O, Hendrickson AE, Curcio CA (1990) Development redistribution of photoreceptors across the Macaca nemestrina (pigtail macaque) retina. J Comp Neurol 298:472-493.

Parr BA, Shea MJ, Vassileva G, McMahon AP (1993) Mouse Wnt genes exhibit discrete domains of expression in the early embryonic CNS and limb buds. Development 119:247-261.

Polans AS, Burton MD, Haley TL, Crabb JW, Palczewski K (1993) Recoverin, but not visinin, is an autoantigen in the human retina identified with a cancer-associated retinopathy. Invest Ophthalmol Vis Sci 34:81-90.

Prada C, Puga J, Perez-Mendez L, Lopez R, Ramirez G (1991) Spatial and temporal patterns of neurogenesis in the chick retina. Eur J Neurosci 3:559-569.

Raymond PA, Barthel LK, Rounsifer ME, Sullivan SA, Knight JK (1993) Expression of rod and cone visual pigments in goldfish and zebrafish: a rhodopsin-like gene is expressed in cones. Neuron 10:1161-1174.

Riddle RD, Johnson RL, Laufer E, Tabin C (1993) Sonic hedgehog mediates the polarizing activity of the ZPA. Cell 75:1401-1416.

Rodieck RW (1973) Structure: general features. In: The vertebrate retina, principles of structure and function (Kennedy D, Park RB, eds), pp 338-368. San Francisco: W. H. Freeman.

Rohlich P, van Veen T, Szel A (1994) Two different visual pigments in one retinal cone cell. Neuron 13:1159-1166.

Saha MS, Grainger RM (1993) Early opsin expression in Xenopus embryos precedes photoreceptor differentiation. Mol Brain Res 17:307-318.

Straznicky C, Chehade M (1987) The formation of the area centralis of the retinal ganglion cell layer in the chick. Development 100:411-420. 
Szel A, Rohlich P, Caffe AR, Juliusson B, Aguirre G, van Veen T (1992) Unique topographic separation of two spectral classes of cones in the mouse retina. J Comp Neurol 325:327-342.

Szel A, Rohlich P, Mieziewska K, Aguirre G, van Veen T (1993) Spatial and temporal differences between the expression of short- and middlewave sensitive cone pigments in the mouse retina: a developmental study. J Comp Neurol 331:564-577.

Szel A, van Veen T, Rohlich P (1994) Retinal cone differentiation [letter]. Nature 370:336.

Takao M, Yasui A, Tokunaga F (1988) Isolation and sequence determination of the chicken rhodopsin gene. Vision Res 28:471-480.

Tokunaga F, Iwasa T, Miyagishi M, Kayada S (1990) Cloning of cDNA and amino acid sequence of one of chicken cone visual pigments. Biochem Biophys Res Commun 173:1212-1217.

Wang SZ, Adler R, Nathans J (1992) A visual pigment from chicken that resembles rhodopsin: amino acid sequence, gene structure, and functional expression. Biochemistry 31:3309-3315.
Wiechmann AF, Hammarback JA (1993) Expression of recoverin mRNA in the human retina: localization by in situ hybridization. Exp Eye Res 57:763-769.

Wikler KC, Rakic P (1991) Rclation of an array of carly-differentiating cones to the photoreceptor mosaic in the primate retina. Nature 351:397-400.

Wikler KC, Rakic P (1994) An array of early differentiating cones precedes the emergence of the photoreceptor mosaic in the fetal monkey retina. Proc Natl Acad Sci USA 91:6534-6538.

Yamagata K, Goto K, Kuo CH, Kondo H, Miki N (1990) Visinin: a novel calcium binding protein expressed in retinal cone cells. Neuron 4:469-476.

Young RW (1985a) Cell differentiation in the retina of the mouse. Anat Rec 212:199-205.

Young RW (1985b) Cell proliferation during postnatal development of the retina in the mouse. Dev Brain Res 21:229-239. 